PROCEEDINGS OF THE

AMERICAN MATHEMATICAL SOCIETY

Volume 134, Number 8, Pages 2213-2222

S 0002-9939(06)08262-1

Article electronically published on February 3, 2006

\title{
BINOMIAL COEFFICIENTS AND QUADRATIC FIELDS
}

\author{
ZHI-WEI SUN
}

(Communicated by Wen-Ching Winnie Li)

\begin{abstract}
Let $E$ be a real quadratic field with discriminant $d \not \equiv 0(\bmod p)$ where $p$ is an odd prime. For $\rho= \pm 1$ we determine $\prod_{0<c<d,\left(\frac{d}{c}\right)=\rho}\left(\begin{array}{c}p-1 \\ \lfloor p c / d\rfloor\end{array}\right)$ modulo $p^{2}$ in terms of a Lucas sequence, the fundamental unit and the class number of $E$.
\end{abstract}

\section{INTRODUCTION}

Let $p$ be an odd prime not dividing a positive integer $m$. A. Granville [G, (1.15)] discovered the remarkable congruence

$$
\prod_{0<k<m}\left(\begin{array}{c}
p-1 \\
\lfloor p k / m\rfloor
\end{array}\right) \equiv(-1)^{(m-1)(p-1) / 2}\left(m^{p}-m+1\right) \quad\left(\bmod p^{2}\right)
$$

where we use $\lfloor x\rfloor$ to denote the integral part of a real number $x$. Subsequently the present author [S1] determined further $\prod_{0<k<m / 2}\left(\begin{array}{c}p-1 \\ \lfloor p k / m\rfloor\end{array}\right) \bmod p^{2}$. In this paper a more sophisticated result connected with real quadratic fields will be established.

For $A, B \in \mathbb{Z}$ the Lucas sequences $u_{n}=u_{n}(A, B)$ and $v_{n}=v_{n}(A, B)(n=$ $0,1,2, \ldots)$ are given by

$$
\begin{aligned}
& u_{0}=0, u_{1}=1, \text { and } u_{n+1}=A u_{n}-B u_{n-1} \text { for } n=1,2,3, \ldots, \\
& v_{0}=2, v_{1}=A, \text { and } v_{n+1}=A v_{n}-B v_{n-1} \text { for } n=1,2,3, \ldots
\end{aligned}
$$

It is well known that

$$
(\alpha-\beta) u_{n}=\alpha^{n}-\beta^{n} \text { and } v_{n}=\alpha^{n}+\beta^{n} \quad \text { for every } n=0,1,2, \ldots,
$$

where $\alpha$ and $\beta$ are the two roots of the equation $x^{2}-A x+B=0$. Also, for any odd prime $p$ we have $u_{p} \equiv\left(\frac{\Delta}{p}\right)(\bmod p)$ and $v_{p} \equiv A(\bmod p)$, where $\Delta=A^{2}-4 B$ and $(\dot{\bar{p}})$ denotes the Legendre symbol. (See, e.g., [R, pp. 41-55].) If $p$ is an odd prime not dividing $B$, then $p \mid u_{p-\left(\frac{\Delta}{p}\right)}$ since $A u_{p}+v_{p}=2 u_{p+1}$ and $A u_{p}-v_{p}=2 B u_{p-1}$.

Throughout this paper, for an assertion $P$ we set

$$
[P]= \begin{cases}1 & \text { if } P \text { holds } \\ 0 & \text { otherwise }\end{cases}
$$

Our main result is as follows.

Received by the editors March 4, 2004 and, in revised form, March 6, 2005.

2000 Mathematics Subject Classification. Primary 11B65; Secondary 11B37, 11B68, 11R11.

The author was supported by the National Science Fund for Distinguished Young Scholars (No. 10425103) and the Key Program of NSF (No. 10331020) in China.

(C)2006 American Mathematical Society Reverts to public domain 28 years from publication 
Theorem 1.1. Let $E$ be a quadratic field with discriminant $d=2^{\alpha} p_{1} \cdots p_{r}$ where $\alpha \in\{0,2,3\}$ and $p_{1}, \ldots, p_{r}$ are distinct odd primes. Let $\varepsilon=(a+b \sqrt{d}) / 2$ be the fundamental unit of the field $E$ where $a, b \in \mathbb{Z}$, and let $N(\varepsilon)$ be the norm $\left(a^{2}-b^{2} d\right) / 4$ of $\varepsilon$ with respect to the field extension $E / \mathbb{Q}$. Let $h$ be the class number of the field $E$, and let $p$ be an odd prime not dividing $d$. Then, for $\rho= \pm 1$ we have

$$
\begin{aligned}
\prod_{\substack{0<c<d \\
\left(\frac{d}{c}\right)=\rho}}\left(\begin{array}{c}
p-1 \\
\lfloor p c / d\rfloor
\end{array}\right) \equiv & 1+\frac{\varphi(d)}{2}\left((\alpha+[\alpha>0])\left(2^{p-1}-1\right)+\sum_{0<i \leq r} \frac{p_{i}^{p}-p_{i}}{p_{i}-1}\right) \\
& +\frac{\rho}{2}\left(\frac{d}{p}\right)^{[N(\varepsilon)=1]} u_{p-\left(\frac{d}{p}\right)}(a, N(\varepsilon)) b d h \quad\left(\bmod p^{2}\right),
\end{aligned}
$$

where $\varphi$ is Euler's totient function and $\left(\frac{d}{.}\right)$ is the Kronecker symbol.

Remark. Under the conditions of Theorem $1.1, d \equiv 1(\bmod 4)$ if $\alpha=0$, and $d / 4 \equiv$ $3(\bmod 4)$ if $\alpha=2$; also $p$ divides $b u_{p-\left(\frac{d}{p}\right)}(a, N(\varepsilon))$, for, if $p \nmid b$ then

$$
\left(\frac{a^{2}-4 N(\varepsilon)}{p}\right)=\left(\frac{b^{2} d}{p}\right)=\left(\frac{d}{p}\right) .
$$

Example. Each of the quadratic fields $\mathbb{Q}(\sqrt{13}), \mathbb{Q}(\sqrt{21}), \mathbb{Q}(\sqrt{6}), \mathbb{Q}(\sqrt{7})$ has class number 1 , and their fundamental units are

$$
\frac{3+\sqrt{13}}{2}, \frac{5+\sqrt{21}}{2}, 5+2 \sqrt{6}=\frac{10+2 \sqrt{24}}{2}, 8+3 \sqrt{7}=\frac{16+3 \sqrt{28}}{2}
$$

with norms $-1,1,1,1$ respectively; see, e.g., [C, p. 271]. Let $p$ be an odd prime and $\rho \in\{1,-1\}$. If $p$ does not divide $13,21,6$, and 7 , respectively, then Theorem 1.1 gives the congruences

$$
\begin{aligned}
& \prod_{\substack{0<c<13 \\
\left(\frac{13}{c}\right)=\rho}}\left(\begin{array}{c}
p-1 \\
\lfloor p c / 13\rfloor
\end{array}\right) \equiv 1+\frac{13^{p}-13}{2}+\rho \frac{13}{2} u_{p-\left(\frac{13}{p}\right)}(3,-1), \\
& \prod_{\substack{0<c<21 \\
\left(\frac{21}{c}\right)=\rho}}\left(\begin{array}{c}
p-1 \\
\lfloor p c / 21\rfloor
\end{array}\right) \equiv 1+3\left(3^{p}-3\right)+7^{p}-7+\rho\left(\frac{21}{p}\right) \frac{21}{2} u_{p-\left(\frac{21}{p}\right)}(5,1), \\
& \prod_{\substack{0<c<24 \\
2 \nmid c,\left(\frac{6}{c}\right)=\rho}}\left(\begin{array}{c}
p-1 \\
\lfloor p c / 24\rfloor
\end{array}\right) \equiv 1+8\left(2^{p}-2\right)+2\left(3^{p}-3\right)+\rho\left(\frac{6}{p}\right) 24 u_{p-\left(\frac{6}{p}\right)}(10,1), \\
& \prod_{\substack{0<c<28 \\
2 \nmid c,\left(\frac{7}{c}\right)=\rho}}\left(\begin{array}{c}
p-1 \\
\lfloor p c / 28\rfloor
\end{array}\right) \equiv 1+9\left(2^{p}-2\right)+7^{p}-7+\rho\left(\frac{7}{p}\right) 42 u_{p-\left(\frac{7}{p}\right)}(16,1)
\end{aligned}
$$

modulo $p^{2}$ respectively, where $\left(\frac{6}{c}\right)$ and $\left(\frac{7}{c}\right)$ are Jacobi symbols.

We deduce Theorem 1.1 by combining the following two theorems.

Theorem 1.2. Let $m>2$ be an integer with the factorization $p_{1}^{\alpha_{1}} \cdots p_{r}^{\alpha_{r}}$ where $p_{1}, \ldots, p_{r}$ are distinct primes and $\alpha_{1}, \ldots, \alpha_{r}$ are positive integers. Let $p$ be an odd 
prime not dividing $m$. Then

$$
\begin{aligned}
& (-1)^{\frac{\varphi(m)}{2} \cdot \frac{p-1}{2}}\left(\frac{p_{1}}{p}\right)^{[r=1]} \prod_{\substack{0<k<m / 2 \\
(k, m)=1}}\left(\begin{array}{c}
p-1 \\
\lfloor p k / m\rfloor
\end{array}\right) \\
\equiv & 1+\frac{\varphi(m)}{2} \sum_{i=1}^{r}\left(\alpha_{i} p_{i}-\alpha_{i}+1\right) \frac{p_{i}^{p-1}-1}{p_{i}-1}\left(\bmod p^{2}\right) .
\end{aligned}
$$

In the next theorem we use the Bernoulli polynomial $B_{n}(x)$ of degree $n$ and the $n$th Bernoulli number $B_{n}=B_{n}(0)$. Also, we let $\mathbb{P}$ denote the set of all (positive) primes.

Theorem 1.3. Let $E$ be a real quadratic field with discriminant $d$ and class number $h$. Let $\varepsilon=(a+b \sqrt{d}) / 2>1$ be the fundamental unit of $E$ where $a, b \in \mathbb{Z}$, and let $N(\varepsilon)$ be the norm $\left(a^{2}-b^{2} d\right) / 4$ of $\varepsilon$. Let $p$ be an odd prime not dividing $d$, and let $u$ stand for $b u_{p-\left(\frac{d}{p}\right)}(a, N(\varepsilon))$. Then

$$
\sum_{c=1}^{d-1}\left(\frac{d}{c}\right)\left(B_{p-1}\left(\frac{c}{d}\right)-B_{p-1}\right) \equiv\left(\frac{d}{p}\right)^{[N(\varepsilon)=-1]} d h \frac{u}{p}(\bmod p),
$$

and

$$
\prod_{\substack{0<c<d / 2 \\
(c, d)=1}}\left(\begin{array}{c}
p-1 \\
\lfloor p c / d\rfloor
\end{array}\right)^{\left(\frac{d}{c}\right)} \equiv \begin{cases}\left(\frac{d}{p}\right)\left(1+\frac{d h u}{2}\right)\left(\bmod p^{2}\right) & \text { if } d=8 \text { or } d \in \mathbb{P} \\
1+\left(\frac{d}{p}\right)^{[N(\varepsilon)=1]} \frac{d h u}{2}\left(\bmod p^{2}\right) & \text { otherwise. }\end{cases}
$$

Remark. In the case where $d \equiv 1(\bmod 4)$ is a prime, $(1.4)$ was proved in GS by means of $p$-adic logarithms and Dirichlet's class number formula (see, e.g., W] $)$.

In the spirit of R. Crandall and C. Pomerance [CP], Theorems 1.1-1.3 might be of computational interest.

We shall make some preparations in the next section and give proofs of Theorems 1.1-1.3 in Section 3.

\section{ON THE SUM $\sum_{\substack{0<k<p \\ m \mid k-r}} \frac{1}{k}$ MODULO $p$}

Bernoulli polynomials play important roles in many aspects. The reader is referred to [IR, pp. 228-248] for their basic properties, and to [DSS for a bibliography of related papers.

In this section we prove the following basic result and derive some consequences.

Theorem 2.1. Let $m$ be a positive integer not divisible by an odd prime $p$. Then for any $r \in \mathbb{Z}$ we have

$$
\sum_{\substack{k=1 \\ k \equiv r(\bmod m)}}^{p-1} \frac{1}{k} \equiv \frac{1}{m}\left(B_{p-1}\left(\left\{\frac{r}{m}\right\}\right)-B_{p-1}\left(\left\{\frac{r-p}{m}\right\}\right)\right)(\bmod p),
$$

where $\{x\}$ stands for the fractional part of a real number $x$. 
Proof. Applying Lemma 3.1 of [S3] with $k=p-2$, we find that

$$
-m \sum_{\substack{j=1 \\ j \equiv r(\bmod m)}}^{p-1} \frac{1}{j} \equiv B_{p-1}\left(\frac{p}{m}+\left\{\frac{r-p}{m}\right\}\right)-B_{p-1}\left(\left\{\frac{r}{m}\right\}\right)(\bmod p) .
$$

For $t=\{(r-p) / m\}$, we have

$$
B_{p-1}\left(\frac{p}{m}+t\right)-B_{p-1}(t)=\sum_{l=1}^{p-1}\left(\begin{array}{c}
p-1 \\
l
\end{array}\right) B_{p-1-l}\left(\left(\frac{p}{m}+t\right)^{l}-t^{l}\right) \equiv 0(\bmod p) .
$$

(Recall that $B_{1}=-1 / 2$ and $B_{2 n+1}=0$ for $n=1,2, \ldots$ Also, $p$ divides no denominators of $B_{0}, B_{2}, \ldots, B_{p-3}$ by the theorem of Clausen and von Staudt (cf. [IR, pp. 233-236]).) Therefore (2.1) follows.

Remark. The author first discovered Theorem 2.1 in Sept. 1991 by using Fourier series, and Lemma 3.1 of [S3] was originally motivated by this result.

Corollary 2.1. Let $m$ and $n$ be positive integers, and let $p$ be an odd prime not dividing $m$. Then

$$
B_{p-1}\left(\left\{\frac{p n}{m}\right\}\right)-B_{p-1} \equiv m \sum_{r=1}^{n} K_{p}(r, m) \equiv-\sum_{\substack{k=1 \\ p \nmid k}}^{\lfloor p n / m\rfloor} \frac{1}{k}(\bmod p),
$$

where

$$
K_{p}(r, m):=\sum_{\substack{k=1 \\ m \mid k-r p}}^{p-1} \frac{1}{k}=\sum_{\substack{l=1 \\ m \mid l-(1-r) p}}^{p-1} \frac{1}{p-l} \equiv-K_{p}(1-r, m)(\bmod p) .
$$

Proof. In view of Theorem 2.1,

$$
\begin{aligned}
m \sum_{r=1}^{n} K_{p}(r, m) & \equiv \sum_{r=1}^{n}\left(B_{p-1}\left(\left\{\frac{r p}{m}\right\}\right)-B_{p-1}\left(\left\{\frac{(r-1) p}{m}\right\}\right)\right) \\
& \equiv B_{p-1}\left(\left\{\frac{p n}{m}\right\}\right)-B_{p-1}(\bmod p) .
\end{aligned}
$$

On the other hand,

$$
-\sum_{r=1}^{n} K_{p}(r, m) \equiv \sum_{r=1}^{n} \sum_{\substack{k=1 \\ m \mid r p-k}}^{p-1} \frac{1}{r p-k}=\sum_{\substack{j=1 \\ p \nmid j, m \mid j}}^{p n} \frac{1}{j}=\sum_{\substack{k=1 \\ p \nmid k}}^{\lfloor p n / m\rfloor} \frac{1}{k m}(\bmod p) .
$$

So we have (2.2).

Let $p$ be an odd prime and let $r$ be any integer. An explicit congruence for $K_{p}(r, 12) \bmod p$ appeared in Corollary 3.3 of [S2]. By Theorem 2.1 and [GS, (4)] we can also determine

$$
K_{p}(3+6 r, 24), K_{p}(5,40), K_{p}(25,40), K_{p}(6,60), K_{p}(36,60)
$$

modulo $p$ in terms of some second-order linear recurrences.

For a prime $p$ and any $a \in \mathbb{Z}$ not divisible by $p$, the Fermat quotient $q_{p}(a)$ is defined as the integer $\left(a^{p-1}-1\right) / p$. 
Corollary 2.2. Let $p$ be an odd prime and let $m$ be a positive integer not divisible by $p$. Then we have

$$
\sum_{r=1}^{m} r K_{p}(r, m) \equiv-q_{p}(m) \quad(\bmod p) .
$$

Proof. By Corollary 2.1,

$$
\begin{aligned}
\sum_{n=1}^{m} \sum_{r=1}^{n} K_{p}(r, m) & \equiv \frac{1}{m} \sum_{n=1}^{m}\left(B_{p-1}\left(\left\{\frac{p n}{m}\right\}\right)-B_{p-1}\right) \\
& \equiv m^{p-2}\left(\sum_{n=1}^{m} B_{p-1}\left(\left\{\frac{p n}{m}\right\}\right)-m B_{p-1}\right) \\
& \equiv \sum_{r=0}^{m-1} m^{p-2} B_{p-1}\left(\frac{r}{m}\right)-m^{p-1} B_{p-1}=\left(1-m^{p-1}\right) B_{p-1}(\bmod p)
\end{aligned}
$$

where we have applied Raabe's theorem in the last step. It is well known that $p B_{p-1} \equiv-1(\bmod p)($ cf. [IR, p. 233]). Also,

$$
\begin{aligned}
& \sum_{n=1}^{m} \sum_{r=1}^{n} K_{p}(r, m)=\sum_{r=1}^{m}(m-(r-1)) K_{p}(r, m) \\
\equiv & -\sum_{r=1}^{m}(m+1-r) K_{p}(m+1-r, m)=-\sum_{s=1}^{m} s K_{p}(s, m)(\bmod p) .
\end{aligned}
$$

So we have (2.4).

Remark. It can be shown that (2.4) is equivalent to a formula of Lerch $\mathrm{L}$ which was deduced in a different way.

\section{Proofs of Theorems $1.1-1.3$}

Proof of Theorem 1.2. For each positive integer $d$ we set

$$
\psi(d)=\prod_{\substack{0<c<d / 2 \\
(c, d)=1}}\left(\begin{array}{c}
p-1 \\
\lfloor p c / d\rfloor
\end{array}\right),
$$

where $\psi(1)$ and $\psi(2)$ are considered as 1 . For any $a \in \mathbb{Z}$ with $p \nmid a$, clearly

$$
\begin{aligned}
a^{p}-a & =a\left(a^{(p-1) / 2}+\left(\frac{a}{p}\right)\right)\left(a^{(p-1) / 2}-\left(\frac{a}{p}\right)\right) \\
& \equiv 2 a\left(\frac{a}{p}\right)\left(a^{(p-1) / 2}-\left(\frac{a}{p}\right)\right)\left(\bmod p^{2}\right) .
\end{aligned}
$$

Thus, Theorem 1.1 of [S1] implies that if $d \not \equiv 0(\bmod p)$ then

$$
\begin{aligned}
& (-1)^{\frac{p-1}{2}\left\lfloor\frac{d-1}{2}\right\rfloor} \prod_{0<c<d / 2}\left(\begin{array}{c}
p-1 \\
\lfloor p c / d\rfloor
\end{array}\right) \\
\equiv & \begin{cases}\left(\frac{d}{p}\right)+\left(\frac{d}{p}\right) \frac{d^{p}-d}{2} & \text { if } 2 \nmid d, \\
\left(\frac{2 d}{p}\right)+\left(\frac{2 d}{p}\right) \frac{d^{p}-d}{2}-\left(\frac{2 d}{p}\right) \frac{2^{p}-2}{2} & \text { if } 2 \mid d,\end{cases} \\
\equiv & \left(\frac{d}{p}\right)\left(\frac{2}{p}\right)^{[2 \mid d]}\left(1+\frac{d^{p}-d}{2}-[2 \mid d]\left(2^{p-1}-1\right)\right)\left(\bmod p^{2}\right) .
\end{aligned}
$$


Since $\prod_{0<k<n / 2}\left(\begin{array}{c}p-1 \\ \lfloor k / n\rfloor\end{array}\right)=\prod_{d \mid n} \psi(d)$ for $n=1,2, \ldots$, applying the Möbius inversion formula we get that

$$
\begin{aligned}
& \psi(m)=\prod_{d \mid m} \prod_{0<c<d / 2}\left(\begin{array}{c}
p-1 \\
\lfloor p c / d\rfloor
\end{array}\right)^{\mu(m / d)} \\
\equiv & (-1)^{\frac{p-1}{2} \sum_{d \mid m} \mu\left(\frac{m}{d}\right)\left(\frac{d-1}{2}-\frac{[2 \mid d]}{2}\right)}\left(\frac{2}{p}\right)^{\sum_{d \mid m} \mu(m / d)[2 \mid d]} \\
& \times \prod_{d \mid m}\left(\frac{d}{p}\right)^{\mu(m / d)} \times \prod_{d \mid m}\left(1+\mu\left(\frac{m}{d}\right)\left(\frac{d^{p}-d}{2}-[2 \mid d]\left(2^{p-1}-1\right)\right)\right)\left(\bmod p^{2}\right) .
\end{aligned}
$$

By elementary number theory, $\sum_{d \mid m} \mu\left(\frac{m}{d}\right) \frac{d-1}{2}=\frac{\varphi(m)}{2}$ and also

$$
\sum_{d \mid m} \mu\left(\frac{m}{d}\right)[2 \mid d]=\sum_{2 c \mid m} \mu\left(\frac{m}{2 c}\right)=[2 \mid m] \sum_{c \mid(m / 2)} \mu\left(\frac{m / 2}{c}\right)=0
$$

since $m>2$. Therefore

$$
(-1)^{\frac{\varphi(m)}{2} \cdot \frac{p-1}{2}} \psi(m) \equiv \prod_{d \mid m}\left(\frac{d}{p}\right)^{\mu(m / d)} \times\left(1+\sum_{d \mid m} \mu\left(\frac{m}{d}\right) \frac{d^{p}-d}{2}\right)\left(\bmod p^{2}\right) .
$$

Observe that

$$
\begin{aligned}
& \prod_{d \mid m}\left(\frac{d}{p}\right)^{\mu(m / d)}=\prod_{I \subseteq\{1, \ldots, r\}}\left(\frac{m / \prod_{i \in I} p_{i}}{p}\right)^{\mu\left(\prod_{i \in I} p_{i}\right)} \\
= & \left(\frac{m^{2^{r}} / \prod_{I \subseteq\{1, \ldots, r\}} \prod_{i \in I} p_{i}}{p}\right)=\left(\frac{m^{2^{r}} / \prod_{i=1}^{r} p_{i}^{2^{r-1}}}{p}\right) \\
= & \left(\frac{\prod_{i=1}^{r} p_{i}^{2^{r-1}\left(2 \alpha_{i}-1\right)}}{p}\right)=\left(\frac{p_{1} \cdots p_{r}}{p}\right)^{2^{r-1}}=\left(\frac{p_{1}}{p}\right)^{[r=1]} .
\end{aligned}
$$

Also,

$$
\begin{aligned}
& \varphi(m)+\sum_{d \mid m} \mu\left(\frac{m}{d}\right)\left(d^{p}-d\right)=\sum_{d \mid m} \mu(d) \frac{m^{p}}{d^{p}}=m^{p} \prod_{i=1}^{r}\left(1-p_{i}^{-p}\right) \\
= & \prod_{i=1}^{r}\left(p_{i}^{\alpha_{i} p}-p_{i}^{\left(\alpha_{i}-1\right) p}\right)=\prod_{i=1}^{r}\left(\left(p_{i}+\left(p_{i}^{p}-p_{i}\right)\right)^{\alpha_{i}}-\left(p_{i}+\left(p_{i}^{p}-p_{i}\right)\right)^{\alpha_{i}-1}\right) \\
\equiv & \prod_{i=1}^{r}\left(p_{i}^{\alpha_{i}}+\alpha_{i} p_{i}^{\alpha_{i}-1}\left(p_{i}^{p}-p_{i}\right)-\left(p_{i}^{\alpha_{i}-1}+\left(\alpha_{i}-1\right) p_{i}^{\alpha_{i}-2}\left(p_{i}^{p}-p_{i}\right)\right)\right) \\
\equiv & \prod_{i=1}^{r}\left(\varphi\left(p_{i}^{\alpha_{i}}\right)+\left(p_{i}^{p-1}-1\right)\left(\alpha_{i} p_{i}^{\alpha_{i}}-\left(\alpha_{i}-1\right) p_{i}^{\alpha_{i}-1}\right)\right) \\
\equiv & \varphi(m)\left(1+\sum_{i=1}^{r} \frac{p_{i}^{p-1}-1}{p_{i}-1}\left(\alpha_{i} p_{i}-\alpha_{i}+1\right)\right)\left(\bmod p^{2}\right) .
\end{aligned}
$$

Thus (1.3) holds in view of the above. 
Proof of Theorem 1.3. Write $\varepsilon^{p-\left(\frac{d}{p}\right)}=(V+U \sqrt{d}) / 2$ where $U, V \in \mathbb{Z}$, and let $p^{\prime}$ be an integer with $p p^{\prime} \equiv 1(\bmod d)$. Theorem 3.1 of Williams [W] states that

$$
h \frac{U}{p} \equiv-\left(\frac{d}{p}\right) N(\varepsilon)^{\left(\left(\frac{d}{p}\right)-1\right) / 2} \sum_{i=1}^{p-1} \frac{\beta_{p}(i)}{i}(\bmod p)
$$

where $\beta_{p}(i)=\sum_{0<j<d\left\{p^{\prime} i / d\right\}}\left(\frac{d}{j}\right)$.

Let $\bar{\varepsilon}=(a-b \sqrt{d}) / 2$. Then $\varepsilon+\bar{\varepsilon}=a$ and $\varepsilon \bar{\varepsilon}=N(\varepsilon)$. Clearly

$$
v_{n}(a, N(\varepsilon))+u_{n}(a, N(\varepsilon)) b \sqrt{d}=\varepsilon^{n}+\bar{\varepsilon}^{n}+\frac{\varepsilon^{n}-\bar{\varepsilon}^{n}}{\varepsilon-\bar{\varepsilon}} b \sqrt{d}=2 \varepsilon^{n}
$$

for $n=0,1, \ldots$; thus $U=b u_{p-\left(\frac{d}{p}\right)}(a, N(\varepsilon))=u\left(\right.$ and $\left.V=v_{p-\left(\frac{d}{p}\right)}(a, N(\varepsilon))\right)$.

Observe that

$$
\begin{aligned}
& \sum_{i=1}^{p-1} \frac{\beta_{p}(i)}{i}=\sum_{j=1}^{d-1}\left(\frac{d}{j}\right) \sum_{\substack{0<i<p \\
d\left\{p^{\prime} i / d\right\}>j}} \frac{1}{i}=\sum_{j=1}^{d-1}\left(\frac{d}{j}\right) \sum_{j<r<d} \sum_{\substack{0<i<p \\
d \mid p^{\prime} i-r}} \frac{1}{i} \\
& =\sum_{j=1}^{d-1}\left(\frac{d}{j}\right) \sum_{j<r<d} \sum_{\substack{0<i<p \\
d \mid i-r p}} \frac{1}{i}=\sum_{j=1}^{d-1}\left(\frac{d}{j}\right) \sum_{j<r<d} K_{p}(r, d) .
\end{aligned}
$$

As $\chi(j)=\left(\frac{d}{j}\right)$ is a nontrivial multiplicative character modulo $d$, the sum $\sum_{j=1}^{d-1}\left(\frac{d}{j}\right)$ vanishes. Therefore, with the help of Corollary 2.1, we have

$$
\begin{aligned}
\sum_{i=1}^{p-1} \frac{\beta_{p}(i)}{i} & =\sum_{j=1}^{d-1}\left(\frac{d}{j}\right)\left(\sum_{r=1}^{d} K_{p}(r, d)-\sum_{r=1}^{j} K_{p}(r, d)\right) \\
& \equiv \sum_{j=1}^{d-1}\left(\frac{d}{j}\right) \frac{1}{d}\left(0-B_{p-1}\left(\left\{\frac{p j}{d}\right\}\right)+B_{p-1}\right) \\
& \equiv-\frac{1}{d}\left(\frac{d}{p}\right) \sum_{j=1}^{d-1}\left(\frac{d}{p j}\right)\left(B_{p-1}\left(\left\{\frac{p j}{d}\right\}\right)-B_{p-1}\right) \\
& \equiv-\frac{1}{d}\left(\frac{d}{p}\right) \sum_{c=1}^{d-1}\left(\frac{d}{c}\right)\left(B_{p-1}\left(\frac{c}{d}\right)-B_{p-1}\right)(\bmod p) .
\end{aligned}
$$

Combining the above we obtain (1.4).

For each $c=1, \ldots, d-1$, we have $\chi(d-c)=\chi(-1) \chi(c)=\chi(c)$ (cf. [C], pp. 35$36])$; also

$$
\begin{aligned}
& (-1)^{\lfloor p c / d\rfloor}\left(\begin{array}{c}
p-1 \\
\lfloor p c / d\rfloor
\end{array}\right)=\prod_{k=1}^{\lfloor p c / d\rfloor}\left(1-\frac{p}{k}\right) \\
\equiv & 1-p \sum_{k=1}^{\lfloor p c / d\rfloor} \frac{1}{k} \equiv 1+p\left(B_{p-1}\left(\left\{\frac{p c}{d}\right\}\right)-B_{p-1}\right)\left(\bmod p^{2}\right)
\end{aligned}
$$


by (2.2). Taking the above congruence and (1.3) modulo $p$, we obtain

$$
\begin{aligned}
\prod_{\substack{0<c<d / 2 \\
(c, d)=1}}(-1)^{\lfloor p c / d\rfloor} & \equiv \prod_{\substack{0<c<d / 2 \\
(c, d)=1}}\left(\begin{array}{c}
p-1 \\
\lfloor p c / d\rfloor
\end{array}\right) \\
& \equiv(-1)^{\frac{\varphi(d)}{2} \cdot \frac{p-1}{2}}\left(\frac{d}{p}\right)^{[d \text { is a prime power }]}(\bmod p)
\end{aligned}
$$

and hence

$$
\prod_{0<c<d / 2}(-1)^{\lfloor p c / d\rfloor\left(\frac{d}{c}\right)}=\left(\frac{d}{p}\right)^{[d=8 \text { or } d \in \mathbb{P}]} .
$$

(Note that $4 \mid \varphi(d)$ and no square of an odd prime divides $d$.) On the other hand,

$$
\begin{aligned}
& \prod_{0<c<d / 2}\left((-1)^{\lfloor p c / d\rfloor}\left(\begin{array}{c}
p-1 \\
\lfloor p c / d\rfloor
\end{array}\right)\right)^{\left(\frac{d}{c}\right)} \\
\equiv & \prod_{0<c<d / 2}\left(1+p\left(\frac{d}{c}\right)\left(B_{p-1}\left(\left\{\frac{p c}{d}\right\}\right)-B_{p-1}\right)\right) \\
\equiv & 1+\frac{p}{2} \sum_{0<c<d / 2}\left(\frac{d}{c}\right)\left(B_{p-1}\left(\left\{\frac{p c}{d}\right\}\right)-B_{p-1}\right) \\
& +\frac{p}{2} \sum_{0<c<d / 2}\left(\frac{d}{d-c}\right)\left(B_{p-1}\left(\left\{\frac{p(d-c)}{d}\right\}\right)-B_{p-1}\right) \\
\equiv & 1+\frac{p}{2} \sum_{c=1}^{d-1}\left(\frac{d}{c}\right)\left(B_{p-1}\left(\left\{\frac{p c}{d}\right\}\right)-B_{p-1}\right) \\
\equiv & 1+\frac{p}{2}\left(\frac{d}{p}\right) \sum_{r=1}^{d-1}\left(\frac{d}{r}\right)\left(B_{p-1}\left(\frac{r}{d}\right)-B_{p-1}\right)\left(\bmod p^{2}\right) .
\end{aligned}
$$

These, together with (1.4), yield

$$
\prod_{0<c<d / 2}\left(\begin{array}{c}
p-1 \\
\lfloor p c / d\rfloor
\end{array}\right)^{\left(\frac{d}{c}\right)} \equiv\left(\frac{d}{p}\right)^{[d=8 \text { or } d \in \mathbb{P}]}\left(1+\frac{d h u}{2}\left(\frac{d}{p}\right)^{[N(\varepsilon)=1]}\right) \quad\left(\bmod p^{2}\right)
$$

It is well known that $N(\varepsilon)=-1$ if $d=8$ or $d \in \mathbb{P}$ (see, e.g., [C, pp. 185-186]). So the desired (1.5) follows.

Proof of Theorem 1.1. By Theorem 1.2 and the proof of Theorem 1.3,

$$
\left(\frac{d}{p}\right)^{[d=8 \text { or } d \in \mathbb{P}]} \prod_{\substack{0<c<d / 2 \\
c, d)=1}}\left(\begin{array}{c}
p-1 \\
\lfloor p c / d\rfloor
\end{array}\right) \equiv 1+\frac{\varphi(d)}{2} F(d, p) \quad\left(\bmod p^{2}\right)
$$

where

$$
\begin{aligned}
F(d, p) & =[\alpha>0](2 \alpha-\alpha+1) \frac{2^{p-1}-1}{2-1}+\sum_{0<i \leq r}\left(p_{i}-1+1\right) \frac{p_{i}^{p-1}-1}{p_{i}-1} \\
& =(\alpha+[\alpha>0])\left(2^{p-1}-1\right)+\sum_{0<i \leq r} \frac{p_{i}^{p}-p_{i}}{p_{i}-1}
\end{aligned}
$$


also

$$
\left(\frac{d}{p}\right)^{[d=8 \text { or } d \in \mathbb{P}]} \prod_{\substack{0<c<d / 2 \\
(c, d)=1}}\left(\begin{array}{c}
p-1 \\
\lfloor p c / d\rfloor
\end{array}\right)^{\left(\frac{d}{c}\right)} \equiv 1+\frac{d h u}{2}\left(\frac{d}{p}\right)^{[N(\varepsilon)=1]}\left(\bmod p^{2}\right)
$$

where $u=b u_{p-\left(\frac{d}{p}\right)}(a, N(\varepsilon)) \equiv 0(\bmod p)$. Therefore

$$
\begin{aligned}
& \prod_{\substack{0<c<d / 2 \\
\left(\frac{d}{c}\right)=\rho}}\left(\begin{array}{c}
p-1 \\
\lfloor p c / d\rfloor
\end{array}\right)\left(\begin{array}{c}
p-1 \\
\lfloor p(d-c) / d\rfloor
\end{array}\right)=\prod_{\substack{0<c<d / 2 \\
(c, d)=1}}\left(\begin{array}{c}
p-1 \\
\lfloor p c / d\rfloor
\end{array}\right)^{1+\rho\left(\frac{d}{c}\right)} \\
\equiv & \left(1+\frac{\varphi(d)}{2} F(d, p)\right)\left(1+\frac{d h u}{2}\left(\frac{d}{p}\right)^{[N(\varepsilon)=1]}\right)^{\rho} \\
\equiv & \left(1+\frac{\varphi(d)}{2} F(d, p)\right)\left(1+\rho \frac{d h u}{2}\left(\frac{d}{p}\right)^{[N(\varepsilon)=1]}\right) \\
\equiv & 1+\frac{\varphi(d)}{2} F(d, p)+\rho \frac{d h u}{2}\left(\frac{d}{p}\right)^{[N(\varepsilon)=1]}\left(\bmod p^{2}\right) .
\end{aligned}
$$

This proves (1.2). We are done.

\section{ACKNOWLEDGMENT}

The author thanks the referee for his many helpful comments.

\section{REFERENCES}

[C] H. Cohn, Advanced Number Theory, Dover Publ. Inc., New York, 1962. MR0594936 (82b:12001)

[CP] R. Crandall and C. Pomerance, Prime Numbers: A Computational Perspective, Springer, New York, 2001. MR1821158 (2002a:11007)

[DSS] K. Dilcher, L. Skula and I. Sh. Slavutskii, Bernoulli numbers, 1713/1990, Queen's Papers in Pure and Appl. Math. 87(1990). The website of the on-line version is http://www . mathstat . dal.ca/dilcher/bernoulli.html. MR/1119305 (92f:11001)

[G] A. Granville, Arithmetic properties of binomial coefficients.I. Binomial coefficients modulo prime powers, in: Organic mathematics (Burnaby, BC, 1995), 253-276, CMS Conf. Proc., 20, Amer. Math. Soc., Providence, RI, 1997. MR1483922 (99h:11016)

[GS] A. Granville and Z. W. Sun, Values of Bernoulli polynomials, Pacific J. Math. 172 (1996), 117-137. MR.1379289 (98b:11018)

[IR] K. Ireland and M. Rosen, A Classical Introduction to Modern Number Theory (Graduate Texts in Math.; 84), 2nd ed., Springer, New York, 1990. MR1070716 (92e:11001)

[L] M. Lerch, Zur Theorie des Fermatschen Quotienten $\left(a^{p-1}-1\right) / p=q(a)$, Math. Ann. 60 (1905), 471-490.

[R] P. Ribenboim, The Book of Prime Number Records, Springer, New York, 1988. MR 0931080 (89e:11052)

[S1] Z. W. Sun, Products of binomial coefficients modulo p2 $p^{2}$ Acta Arith. 97 (2001), 87-98. MR 1819624 (2002m:11013)

[S2] Z. W. Sun, On the sum $\sum_{k \equiv r}(\bmod m)\left(\begin{array}{l}n \\ k\end{array}\right)$ and related congruences, Israel J. Math. 128 (2002), 135-156. MR 1910378 (2003d:11026) 
[S3] Z. W. Sun, General congruences for Bernoulli polynomials, Discrete Math. 262 (2003), 253-276. MR.1951393 (2003m:11037)

[W] H. C. Williams, Some formulae concerning the fundamental unit of a real quadratic field, Discrete Math. 92 (1991), 431-440. MR.1140604 (92j:11126)

Department of Mathematics, Nanjing University, Nanjing 210093, People's Republic OF CHINA

E-mail address: zwsun@nju.edu.cn 